\title{
Teleophthalmology and its evolving role in a COVID-19 pandemic: A scoping review
}

Jiamin Charmaine Chong, ${ }^{1}$ MBBS, Chai-Hoon Nowel $\underline{\text { Tan }},{ }^{1}$ MBBS, David Z Chen,,${ }^{1,2}$ MBBS, MMed (Ophth), FRCOphth

\begin{abstract}
Introduction: Teleophthalmology may assist the healthcare sector in adapting to limitations imposed on clinical practice by a viral pandemic. A scoping review is performed in this study to assess the current applications of teleophthalmology for its suitability to diagnose, monitor or manage ophthalmological conditions with accuracy.

Methods: A search of PubMed was conducted for teleophthalmology-related articles published from 1 January 2018 to 4 May 2020. Only articles that focused on the use of teleophthalmology in terms of diagnosis and management, as well as its benefits and detriments, were included. The Mixed Methods Appraisal Tool (MMAT) was used to assess the quality of the included articles.

Results: A total of 38 articles were assessed at the full-text level. There were 2 qualitative studies and 1 quantitative randomised controlled trial, while the majority were either quantitative descriptive studies $(19,50.0 \%)$ or quantitative non-randomised studies $(16,42.1 \%)$. Overall, 8 studies described reducing manpower requirements, 4 described reducing direct patient-doctor contact, 17 described storage of medical imaging and clinical data, and 9 described real-time teleconferencing. The MMAT analysis revealed limitations in appropriate sampling strategy in both quantitative non-randomised studies ( 9 of $16,56.3 \%$ ) and quantitative descriptive studies ( 9 of 19, 47.4\%). Cost-effectiveness of teleophthalmology was not performed in any included study.

Conclusion: This current review of the various aspects of teleophthalmology describes how it may potentially assist the healthcare sector to cope with the limitations imposed by a viral pandemic through technology. Further research is required to evaluate the cost-effectiveness of the various strategies.
\end{abstract}

\section{Ann Acad Med Singap 2021;50:61-76}

Keywords: Artificial intelligence, health informatics, ophthalmology, teleconsultation, telemedicine

\section{INTRODUCTION}

The World Health Organization declared coronavirus disease 2019 (COVID-19) a Public Health Emergency of International Concern on 30 January $2020 .^{1}$

The virus has a potentially long incubation period of beyond 14 days, ${ }^{2}$ with ease of human-to-human transmission. ${ }^{3}$ To control the outbreak, many countries have implemented nationwide lockdowns and social distancing measures, which have brought challenges to accessibility of healthcare services and continuation of long-term medical care, necessitating consideration of alternatives such as remote teleconsultations. As more healthcare workers are shunted to the frontlines to deal with increasing numbers of COVID-19 patients, reduced manpower in other specialties such as ophthalmology may cause limitations to working capacity. Telemedicine may provide solutions mitigating the disruptive effect COVID-19 has on the current model of patient care. ${ }^{4}$

Ophthalmology may be a specialty particularly vulnerable to COVID-19 transmission. It has been suggested that ocular signs and symptoms may precede the appearance of respiratory symptoms. ${ }^{5}$ Therefore, it is plausible that the first contact with an undiagnosed COVID-19 patient may happen within the ophthalmology clinic. Furthermore, ophthalmologists are traditionally reliant on physical examination of patients within close proximity for diagnosis and management. ${ }^{6}$ Ophthalmology instruments require their operators to

\footnotetext{
${ }^{1}$ Yong Loo Lin School of Medicine, National University of Singapore, Singapore

${ }^{2}$ Department of Ophthalmology, National University Hospital, Singapore

Correspondence: Dr David Chen, Department of Ophthalmology, National University Hospital, 1E Kent Ridge Road, NUHS Tower Block, Level 7, Singapore 119228.

Email: david_chen@nuhs.edu.sg
} 
be within the radius of droplet spread of up to $6 \mathrm{~m}$, and procedures such as air pressure tonometry and lacrimal irrigation may result in micro-aerosolisation of ocular surface microbes. ${ }^{7}$ Hence examiners are put at risk should the patients examined be COVID-19-positive. The risks of micro-aerosolisation of ocular surface microbes are compounded because of the high-volume nature of ophthalmology clinics, which may preclude thorough disinfection between patients. ${ }^{8}$ Although barrier techniques may be introduced to mitigate such risks, they nevertheless impede gold standards of physical examination using such traditional instruments. ${ }^{9}$

With advancements in medical technologies and telecommunications infrastructure, the integration of telemedicine services shows great potential in supporting healthcare, particularly when distance separates key stakeholders-patients and doctors. ${ }^{10,11}$ Teleophthalmology has been widely studied in terms of screening by primary care physicians, ${ }^{12}$ virtual diagnostic consultations with specialists ${ }^{13}$ and treatment planning for disease management. ${ }^{14}$ Furthermore, there have been documentations highlighting its successful application when tailored to multiple conditions including diabetic retinopathy and glaucoma. ${ }^{15,16}$ Ostensibly, teleophthalmology shows great potential to meet healthcare limitations brought forth by the pandemic, and allows essential care provision to continue with minimal disruption. However, it is currently unclear what kind of information is available regarding the role of teleophthalmology in diagnosing, monitoring, or managing ophthalmological conditions.

This article will perform a scoping review to systemically map the various methods of teleophthalmology trialled, and how these can be further explored and adapted to overcome the practical limitations imposed by a viral pandemic.

\section{METHODS}

A search of PubMed was conducted on 4 May 2020 for studies and literature reviews relating telemedicine to its practice in teleophthalmology according to the Preferred Reporting Items for Systematic Reviews and Meta-Analyses Extension for Scoping Reviews (PRISMA-ScR) guidelines. ${ }^{17}$ The following search strategy was used: "telemedicine" (MeSH Terms) OR "telemedicine" (All Fields) AND "ophthalmology" (MeSH Terms) OR "ophthalmology" (All Fields). As telemedicine evolves with rapid advances in technology, the search criteria were restricted to articles published from 1 January 2018 to 4 May 2020. Articles unavailable in English were excluded. Duplicate articles were manually excluded by 2 reviewers in the team by comparing article titles, names of authors, years and publication journal names.

Studies were included if all of the following were fulfilled: (1) use of telemedicine in the workflow; (2) telemedicine used in relation to diagnosing, monitoring or managing ophthalmological conditions; (3) the benefits and detriments of existing teleophthalmology practices were reviewed; and (4) the article compared accuracy of teleophthalmology tools used to diagnose or grade severity of ophthalmological conditions, against the gold standard of in-person patient review by ophthalmologists.

Studies were excluded if the article did not focus on the clinical benefits or detriments of teleophthalmology. The 2 reviewers independently examined the titles and abstracts of results from the database search, including articles according to the eligibility criteria. Whenever the initial review based on the abstracts was inconclusive, the full text of the articles was read to determine if the eligibility criteria were met. Any conflict regarding inclusion was resolved by consensus discussion, or through discussion with a third reviewer in the team. All included titles were then independently read in full text.

Given the heterogeneity of articles expected, quantitative analysis was not performed. Instead, the Mixed Methods Appraisal Tool (MMAT) was used to assess the quality of the included articles. ${ }^{18}$ The MMAT is developed to appraise the methodological quality of 5 categories of studies: qualitative research, randomised controlled trials (RCTs), non-randomised studies, quantitative descriptive studies, and mixed methods studies. It comprises 2 screening questions, followed by 5 criteria in each of the appropriate category of studies to appraise. Two researchers in the team independently scored each article according to the MMAT, and any disagreement was resolved through discussion.

\section{RESULTS}

\section{Study characteristics of included studies}

The initial database search yielded a total of 781 articles after including 28 primary sources of relevant review articles, of which 38 studies were eventually included in our qualitative synthesis (Fig. 1). Of the 38 included studies, 19 studies $(50.0 \%)$ were categorised as quantitative descriptive studies and $16(42.1 \%)$ quantitative non-randomised studies. One study $(2.6 \%)$ was included as an RCT, and 2 other studies (5.3\%) were qualitative studies.

In view of the heterogeneity of studies included, a summary describing the study design, main teleophthalmology feature and primary outcome 


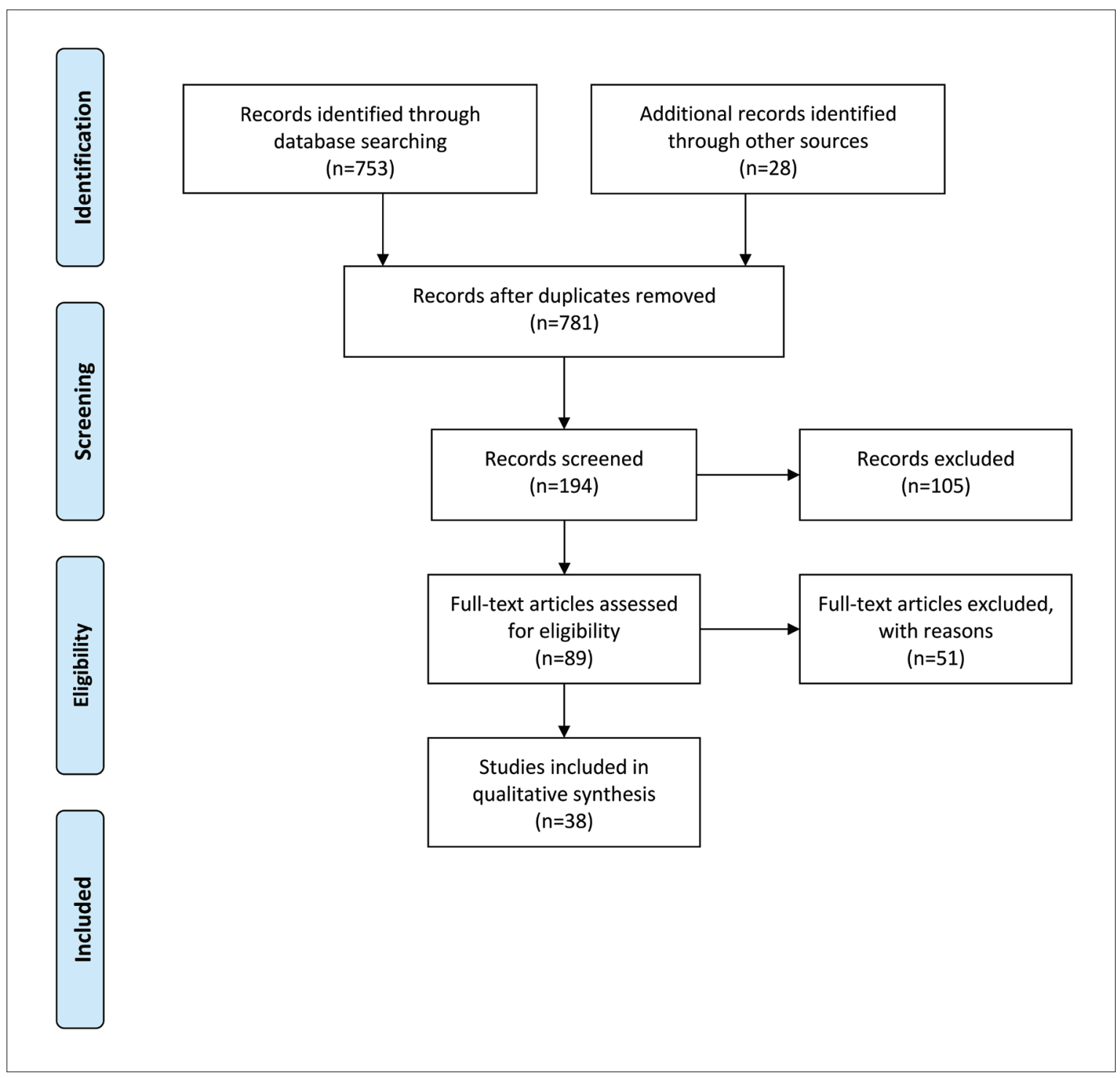

Fig. 1. PRISMA 2009 flow diagram.

Moher D, Liberati A, Tetzlaff J, et al. Preferred Reporting Items for Systematic Reviews and Meta-Analyses: The PRISMA Statement. PLoS Med 2009;6:e1000097.

measure is shown in Table 1. Of the 38 studies, 28 (73.7\%) described asynchronous teleconsultation. Focus was on diagnosis in 15 studies (39.5\%), on referral after screening in $14(36.8 \%)$ and on management in the remaining $9(23.7 \%)$. The primary outcome measure was clinical effectiveness (e.g. accurate diagnosis, appropriate management) in 26 studies $(68.4 \%)$, operational efficiency (e.g. reduced manpower, increased healthcare provision coverage) in $7(18.4 \%)$ and patient-related outcomes (satisfaction, compliance) in $5(13.2 \%)$. No study reported cost-effectiveness or cost savings. Detailed study characteristics and findings are elaborated in Table 2.

\section{Critical appraisal of included studies using MMAT}

The MMAT appraisal questions specific to study types were applied and are summarised in Table 3. For the only RCT included in this study, we found that the approach to cluster randomisation by clinic, instead of by individual patients, to be a limitation. The outcome assessors were also not masked. Of the 16 quantitative non-randomised studies, more than half included participants who were not representative of the target population $(9,56.3 \%)$, while the other criteria were met by most studies; all studies, however, reported good adherence to intervention $(16,100 \%)$. Of the 19 quantitative descriptive studies, limitations were noted 
Table 1. Summary of included studies

\begin{tabular}{|c|c|c|c|}
\hline Author & Study design & Main teleophthalmology feature & Primary outcome \\
\hline Daruich A et al. ${ }^{19}$ & Qualitative study & Synchronous teleconsultation (diagnosis) & Clinical effectiveness \\
\hline Liu Y et al. ${ }^{20}$ & Qualitative study & Asynchronous teleconsultation (referral) & Patient satisfaction \\
\hline Joseph S et al..$^{21}$ & Quantitative RCT & Asynchronous teleconsultation (diagnosis) & Clinical effectiveness \\
\hline Laurent $\mathrm{C}$ et al. ${ }^{22}$ & Quantitative non-randomised study & Asynchronous teleconsultation (diagnosis) & Clinical effectiveness \\
\hline Wisse RPL et al. ${ }^{24}$ & Quantitative non-randomised study & Synchronous teleconsultation (diagnosis) & Clinical effectiveness \\
\hline Maa AY et al. ${ }^{25}$ & Quantitative non-randomised study & Asynchronous teleconsultation (referral) & Clinical effectiveness \\
\hline Roelofs $\mathrm{K}$ et al. ${ }^{26}$ & Quantitative non-randomised study & Asynchronous teleconsultation (diagnosis) & Clinical effectiveness \\
\hline Chandrasekaran S et al. ${ }^{27}$ & Quantitative non-randomised study & Asynchronous teleconsultation (diagnosis) & Clinical effectiveness \\
\hline Phanphruk W et al. ${ }^{28}$ & Quantitative non-randomised study & Asynchronous teleconsultation (management) & Clinical effectiveness \\
\hline Tsapakis S et al. ${ }^{29}$ & Quantitative non-randomised study & Asynchronous teleconsultation (management) & Clinical effectiveness \\
\hline Young K et al. ${ }^{30}$ & Quantitative non-randomised study & Synchronous teleconsultation (management) & Operational efficiency \\
\hline Lapere $\mathrm{S}$ et al. ${ }^{31}$ & Quantitative non-randomised study & Synchronous teleconsultation (management) & Clinical effectiveness \\
\hline Gonzalez $\mathrm{F}$ et al. ${ }^{32}$ & Quantitative non-randomised study & Synchronous teleconsultation (diagnosis) & Operational efficiency \\
\hline Bursztyn L et al. ${ }^{33}$ & Quantitative non-randomised study & Asynchronous teleconsultation (diagnosis) & Clinical effectiveness \\
\hline Maka E et al. ${ }^{34}$ & Quantitative non-randomised study & Asynchronous teleconsultation (diagnosis) & Clinical effectiveness \\
\hline Hadziahmetovic $\mathrm{M}$ et al. ${ }^{35}$ & Quantitative non-randomised study & Asynchronous teleconsultation (referral) & Clinical effectiveness \\
\hline Schallhorn SC et al. ${ }^{36}$ & Quantitative non-randomised study & Synchronous teleconsultation (management) & Clinical effectiveness \\
\hline Odden JL et al. ${ }^{37}$ & Quantitative non-randomised study & Asynchronous teleconsultation (management) & Clinical effectiveness \\
\hline Bartnik SE et al. ${ }^{13}$ & Quantitative descriptive study & Synchronous teleconsultation (diagnosis) & Clinical effectiveness \\
\hline Starr MR et al..$^{39}$ & Quantitative descriptive study & Synchronous teleconsultation (management) & Clinical effectiveness \\
\hline Hark LA et al. ${ }^{40}$ & Quantitative descriptive study & Asynchronous teleconsultation (referral) & Clinical effectiveness \\
\hline Bittner AK et al. ${ }^{41}$ & Quantitative descriptive study & Synchronous teleconsultation (management) & Patient satisfaction \\
\hline Hark LA et al. ${ }^{42}$ & Quantitative descriptive study & Asynchronous teleconsultation (referral) & Clinical effectiveness \\
\hline Yaslam M et al. ${ }^{15}$ & Quantitative descriptive study & Asynchronous teleconsultation (diagnosis) & Clinical effectiveness \\
\hline Giorgis AT et al. ${ }^{16}$ & Quantitative descriptive study & Asynchronous teleconsultation (diagnosis) & Clinical effectiveness \\
\hline Safi $\mathrm{S}$ et al. ${ }^{43}$ & Quantitative descriptive study & Asynchronous teleconsultation (referral) & Clinical effectiveness \\
\hline Modjtahedi BS et al. ${ }^{44}$ & Quantitative descriptive study & Asynchronous teleconsultation (referral) & Patient satisfaction \\
\hline Kortuem K et al. ${ }^{45}$ & Quantitative descriptive study & Asynchronous teleconsultation (referral) & Operational efficiency \\
\hline Host BK et al. ${ }^{46}$ & Quantitative descriptive study & Synchronous teleconsultation (diagnosis) & Patient satisfaction \\
\hline Mastropasqua L et al..$^{47}$ & Quantitative descriptive study & Asynchronous teleconsultation (diagnosis) & Clinical effectiveness \\
\hline Strul S et al. ${ }^{48}$ & Quantitative descriptive study & Asynchronous teleconsultation (referral) & Operational efficiency \\
\hline Martinez JA et al. ${ }^{49}$ & Quantitative descriptive study & Asynchronous teleconsultation (referral) & Patient compliance \\
\hline Avendaño-Veloso A et al. ${ }^{50}$ & Quantitative descriptive study & Asynchronous teleconsultation (referral) & Operational efficiency \\
\hline Grau E et al. ${ }^{51}$ & Quantitative descriptive study & Asynchronous teleconsultation (referral) & Clinical effectiveness \\
\hline Kern $\mathrm{C}$ et al. ${ }^{52}$ & Quantitative descriptive study & Asynchronous teleconsultation (referral) & Operational efficiency \\
\hline Afshar AR et al. ${ }^{53}$ & Quantitative descriptive study & Asynchronous teleconsultation (referral) & Clinical effectiveness \\
\hline
\end{tabular}

RCT: randomised controlled trial

Superscript numbers: Refer to References 


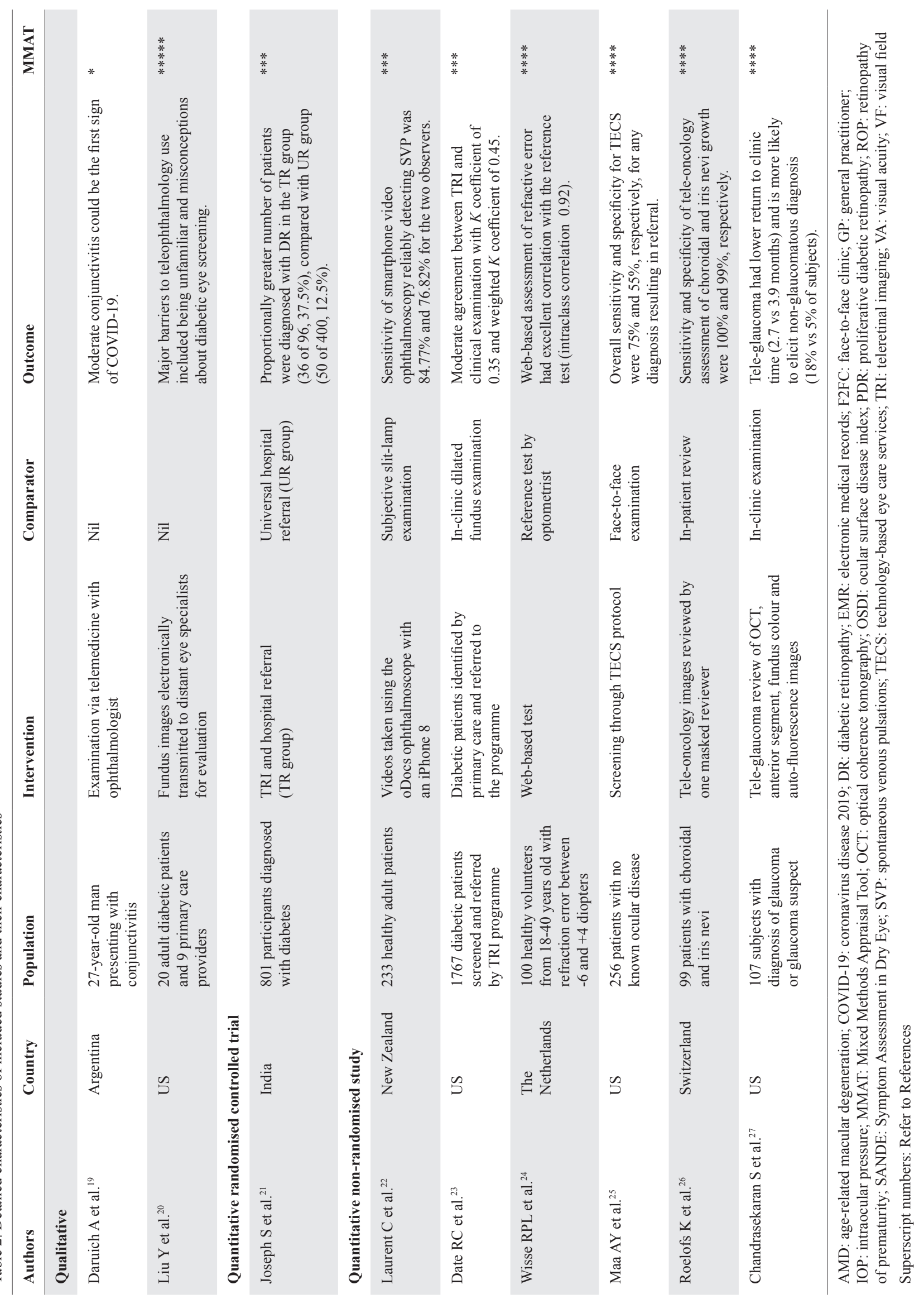




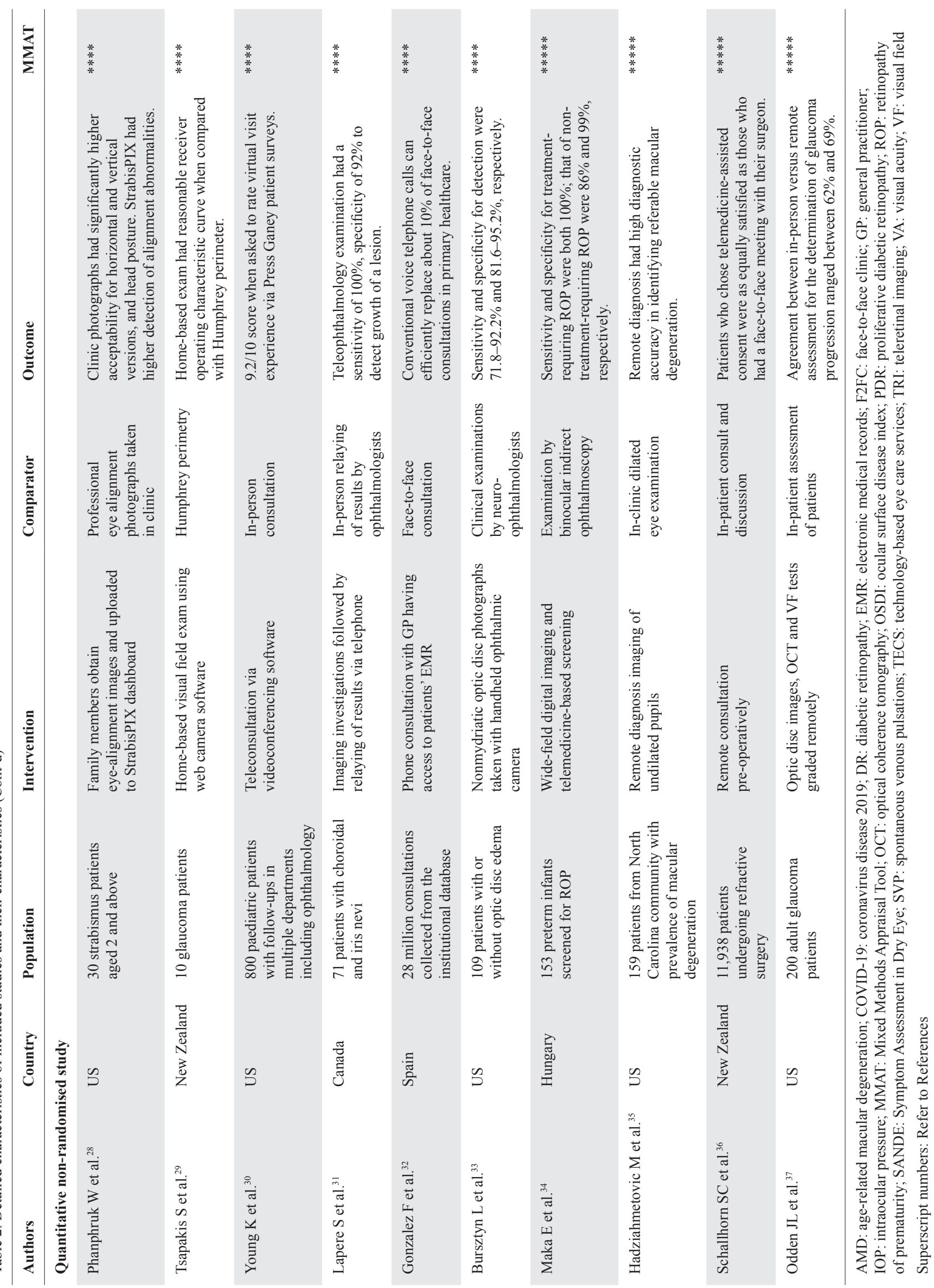




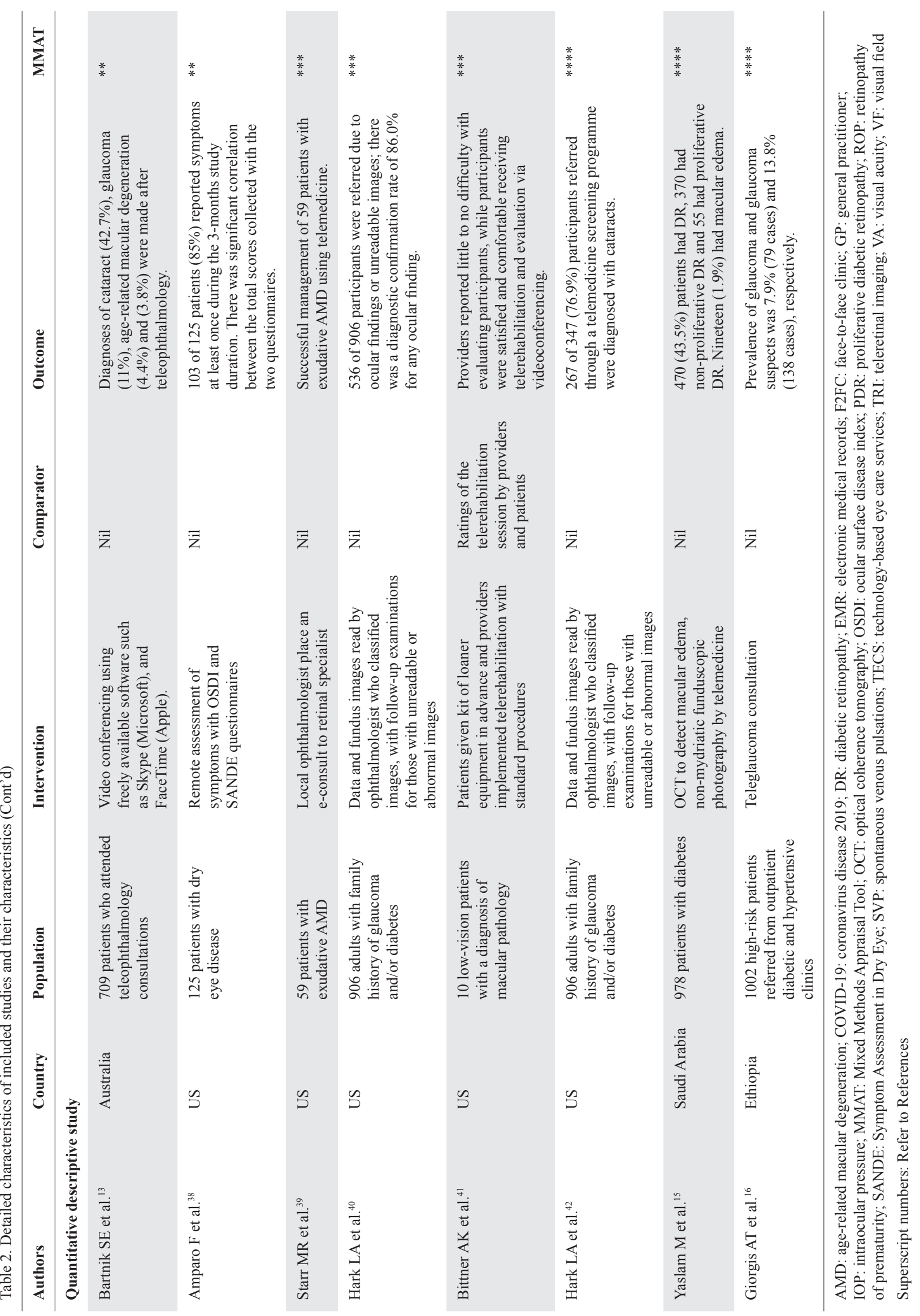




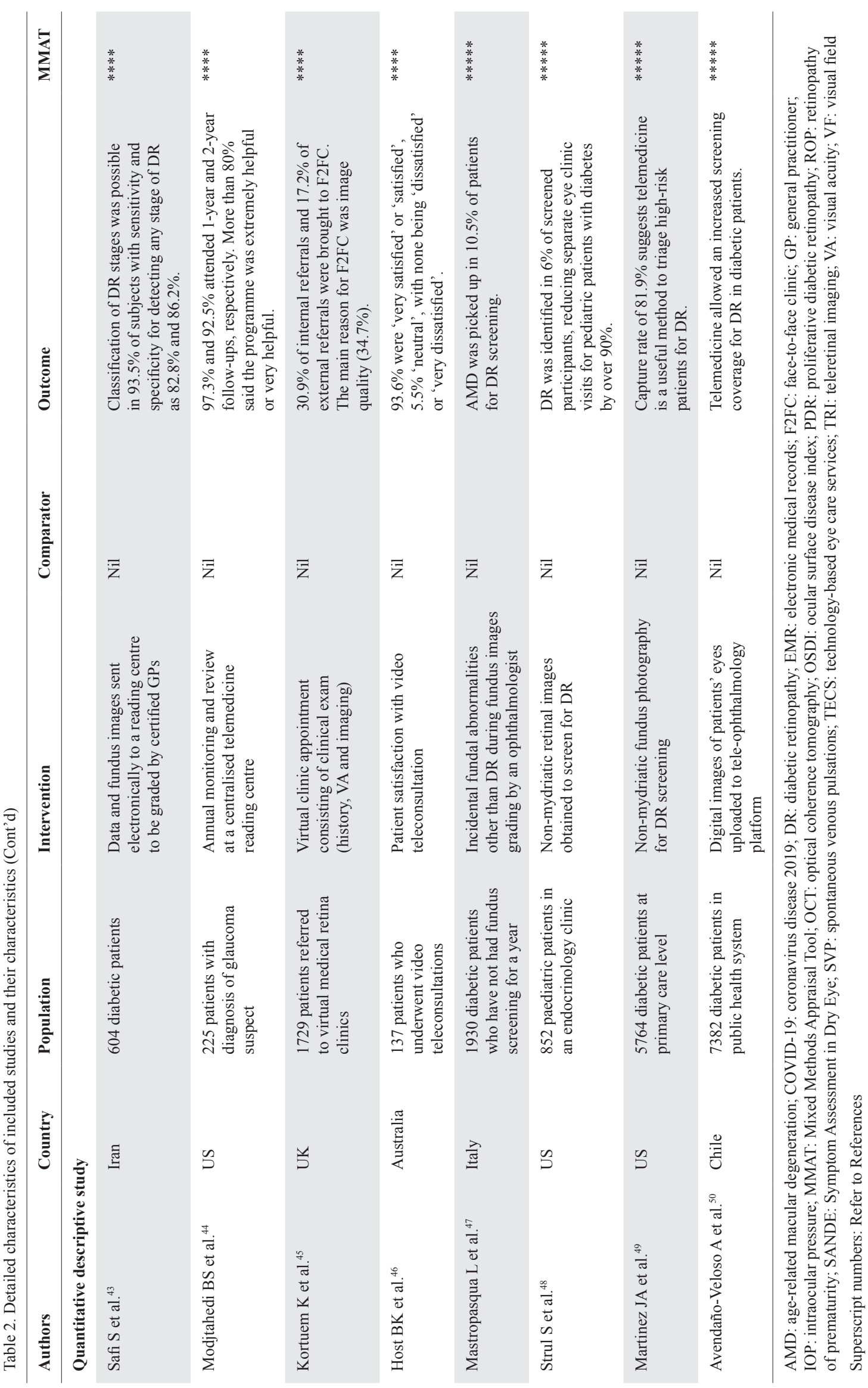




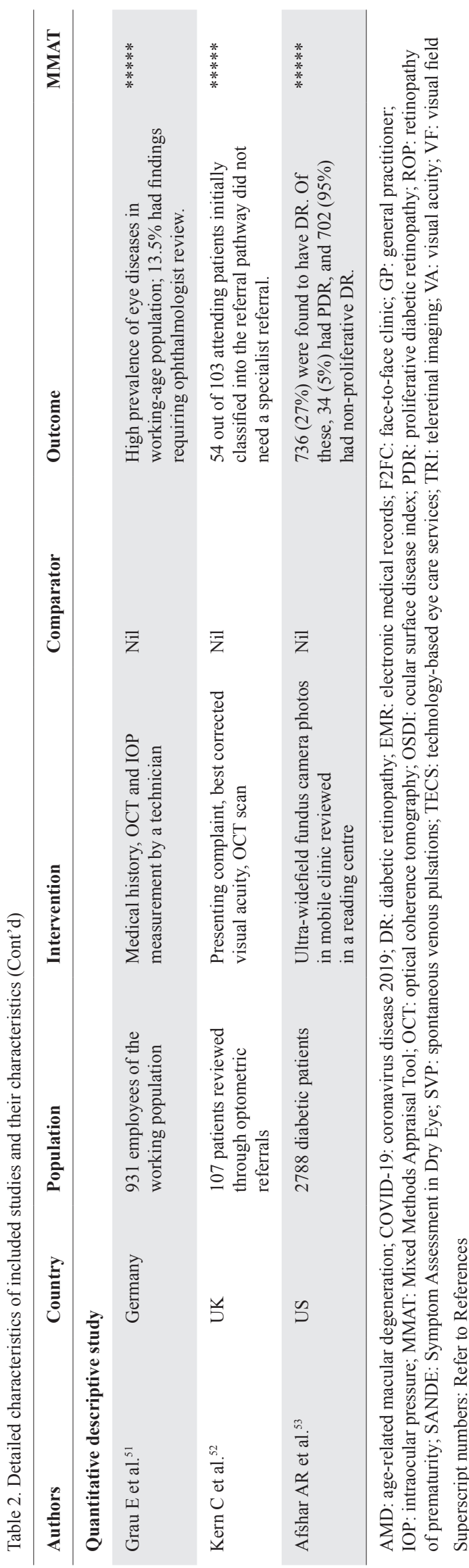

in sampling strategy $(4,21.1 \%)$, sample population $(6,31.6 \%)$ and statistical analysis $(5,26.3 \%)$, while the outcome measures were deemed appropriate for most of the studies $(18,94.7 \%)$.

Overall, the studies focused on 4 main applicable qualities of teleophthalmology: (1) reducing manpower requirements in outpatient setting; (2) reducing direct patient-doctor contact requirements; (3) storage of medical imaging and clinical data for more time-efficient review; and (4) real-time videoconferencing and realtime transmission of diagnostics.

\section{DISCUSSION}

\section{Reducing manpower requirements in the outpatient setting}

In this COVID-19 pandemic, manpower shortages in the frontline and the overworking of healthcare worker have been a major talking point. ${ }^{54}$ Consultants from different specialties may be transferred to the frontline, such as to the intensive care unit and infectious disease wards, to make up for the staffing shortage. In response, there has been an increasing shift in focus to telemedicine in order to provide continuous necessary patient care in other specialisations. ${ }^{4}$ In ophthalmology, the use of telemedicine for screening and monitoring of common eye diseases at the primary care level indicates possibilities of more selective specialists referrals. ${ }^{21,25,51}$

Screening for basic ocular conditions by primary care physicians or technicians ${ }^{27,53}$ via the use of teleophthalmology may help reduce the specialists' workload. ${ }^{16,27,50,51,53}$ For instance, a teleophthalmology screening initiative involving 256 patients yielded substantial agreement for common ocular conditions when compared with face-to-face examination. ${ }^{25}$ Patients could therefore be redirected to the primary care team to reduce unnecessary referrals to tertiary hospitals, saving time for both the physicians and patients. Teleophthalmology has enabled primary care physicians to shoulder the specialists' workload and cut down referral numbers.

Virtual ophthalmology clinics form another avenue of maximising clinic efficiency. ${ }^{31,38,39}$ In Moorfields Eye Hospital, London, a virtual medical retina clinic appointment included ocular imaging followed by a holistic virtual clinical examination, and enabled each doctor to attend to more patients over a fixed time..$^{45}$

\section{Reducing direct patient-doctor contact requirements}

The development of mobile, web-based and similar tools could help minimise transmission risks of COVID-19 during ophthalmology consultations by providing 
Table 3. Application of MMAT appraisal questions

\begin{tabular}{|c|c|c|c|c|c|}
\hline Authors & Question 1 & Question 2 & Question 3 & Question 4 & Question 5 \\
\hline \multicolumn{6}{|l|}{ Qualitative } \\
\hline Daruich A et al. ${ }^{19}$ & 0 & 0 & 0 & 1 & 0 \\
\hline Liu Y et al. ${ }^{20}$ & 1 & 1 & 1 & 1 & 1 \\
\hline \multicolumn{6}{|c|}{ Quantitative randomised controlled trial } \\
\hline Joseph S et al. ${ }^{21}$ & 0 & 1 & 1 & 0 & 1 \\
\hline \multicolumn{6}{|c|}{ Quantitative non-randomised study } \\
\hline Laurent $\mathrm{C}$ et al..$^{22}$ & 0 & 0 & 1 & 1 & 1 \\
\hline Date $\mathrm{RC}$ et al. ${ }^{23}$ & 0 & 1 & 0 & 1 & 1 \\
\hline Wisse RPL et al. ${ }^{24}$ & 0 & 1 & 1 & 1 & 1 \\
\hline Maa AY et al. ${ }^{25}$ & 0 & 1 & 1 & 1 & 1 \\
\hline Chandrasekaran S et al. ${ }^{27}$ & 1 & 1 & 1 & 0 & 1 \\
\hline Phanphruk W et al. ${ }^{28}$ & 0 & 1 & 1 & 1 & 1 \\
\hline Tsapakis S et al. ${ }^{29}$ & 0 & 1 & 1 & 1 & 1 \\
\hline Young $\mathrm{K}$ et al. ${ }^{30}$ & 0 & 1 & 1 & 1 & 1 \\
\hline Lapere $\mathrm{S}$ et al. ${ }^{31}$ & 1 & 1 & 1 & 0 & 1 \\
\hline Gonzalez $\mathrm{F}$ et al. ${ }^{32}$ & 0 & 1 & 1 & 1 & 1 \\
\hline Bursztyn L et al. ${ }^{33}$ & 0 & 1 & 1 & 1 & 1 \\
\hline Maka E et al. ${ }^{34}$ & 1 & 1 & 1 & 1 & 1 \\
\hline Hadziahmetovic $\mathrm{M}$ et al. ${ }^{35}$ & 1 & 1 & 1 & 1 & 1 \\
\hline Schallhorn SC et al. ${ }^{36}$ & 1 & 1 & 1 & 1 & 1 \\
\hline Odden JL et al..$^{37}$ & 1 & 1 & 1 & 1 & 1 \\
\hline Bartnik SE et al. ${ }^{13}$ & 0 & 0 & 0 & 1 & 1 \\
\hline Amparo $\mathrm{F}$ et al. ${ }^{38}$ & 0 & 0 & 1 & 0 & 1 \\
\hline Starr MR et al. ${ }^{39}$ & 0 & 1 & 1 & 0 & 1 \\
\hline Hark LA et al. ${ }^{40}$ & 1 & 0 & 1 & 1 & 0 \\
\hline Bittner AK et al. ${ }^{41}$ & 0 & 1 & 1 & 1 & 0 \\
\hline Hark LA et al. ${ }^{42}$ & 1 & 1 & 1 & 0 & 1 \\
\hline Yaslam M et al. ${ }^{15}$ & 1 & 0 & 1 & 1 & 1 \\
\hline Giorgis AT et al. ${ }^{16}$ & 1 & 1 & 1 & 1 & 0 \\
\hline Safi S et al. ${ }^{43}$ & 1 & 0 & 1 & 1 & 1 \\
\hline Modjtahedi BS et al. ${ }^{44}$ & 1 & 1 & 1 & 1 & 0 \\
\hline Kortuem K et al. ${ }^{45}$ & 1 & 1 & 1 & 1 & 0 \\
\hline Host BK et al. ${ }^{46}$ & 1 & 0 & 1 & 1 & 1 \\
\hline Mastropasqua L et al. ${ }^{47}$ & 1 & 1 & 1 & 1 & 1 \\
\hline Strul S et al. ${ }^{48}$ & 1 & 1 & 1 & 1 & 1 \\
\hline Martinez JA et al. ${ }^{49}$ & 1 & 1 & 1 & 1 & 1 \\
\hline Avendaño-Veloso A et al. ${ }^{50}$ & 1 & 1 & 1 & 1 & 1 \\
\hline Grau E et al..$^{51}$ & 1 & 1 & 1 & 1 & 1 \\
\hline Kern $\mathrm{C}$ et al..$^{52}$ & 1 & 1 & 1 & 1 & 1 \\
\hline Afshar AR et al..$^{53}$ & 1 & 1 & 1 & 1 & 1 \\
\hline
\end{tabular}

Superscript numbers: Refer to References 
more socially distant alternatives to close-proximity physical examinations. Wisse et al. trialled a web-based assessment of refractive error that was excellently correlated with reference tests of uncorrected distance visual acuity performed by optometrists. ${ }^{24}$ This Easee assessment can be taken by a patient autonomously on a smartphone or computer, reducing or eliminating entirely clinician-patient proximity during the measurement.

Many studies have highlighted the utility of remote fundus imaging through modified portable nonmydriatic fundus cameras in identifying posterior pole pathologies. ${ }^{15,33,55}$ Bursztyn et al. reported that fundus images taken by minimally trained non-physicians met threshold sensitivity and specificity when remotely graded by fellowship-trained neuro-ophthalmologists. ${ }^{33}$ Separately, smartphone video ophthalmoscopy could also identify fundus details including spontaneous venous pulsation. ${ }^{22} \mathrm{~A}$ possibility hence exists for nonophthalmic staff in COVID-19 wards to use portable equipment to screen for potentially sight-threatening fundus pathology in the absence of an ophthalmologist.

Teleophthalmology also has the potential to remodel ophthalmic investigations. The rise of home-based perimetry provides an alternative for bulky, non-portable perimetry devices. Tsapakis et al. demonstrated that home-based visual field testing using smartphone was comparable to Humphrey perimeter testing, with good diagnostic accuracy in the measurement of ocular alignment. ${ }^{29}$ On the other hand, the StrabisPIX smartphone application could detect abnormalities in head posture and versions at a similar rate to clinic photographs, while having significantly higher detection rates of alignment abnormalities. ${ }^{28}$ Thus, smartphone applications and related home-based tests are feasible adjuncts to teleophthalmology consults.

\section{Storage of medical imaging and clinical data for more time-efficient review}

In the "store-and-forward" (i.e. asynchronous) method of data capture, data are obtained from patients - typically patient histories or photographs - stored in a secure online platform and forwarded to the specialist for subsequent review. The majority of store-and-forward teleophthalmology utilises image data, although some authors have demonstrated the utility of pre-recorded video ophthalmoscopy in detecting dynamic fundus changes. ${ }^{22}$ Remote specialists may find video recordings to be more representative of clinical assessment, while affording some flexibility in time of review. Nonetheless, some false positives were reported when grading said smartphone videos, and the use of smartphone video ophthalmoscopy may be limited to initial screening rather than formal consultation. While the remainder of this section focuses on the benefits of the store-andforward of image data, it should be noted that further gains are likely to accrue by exploring alternative data mediums such as video.

Several key advantages of the store-and-forward method when employed to diagnose glaucoma, diabetic retinopathy and macular degeneration are discussed as follows.

\section{Glaucoma}

In a longitudinal study on glaucoma suspect monitoring via telemedicine in which the patients' results attained at local eye clinics were sent to a centralised reading centre, ease of access to care with telemedicine showed higher adherence rates to follow-up. ${ }^{44}$ Therefore, storeand-forward systems reduce loss-to-follow-up rates. ${ }^{44,48}$ Given that many patients are unable to physically attend clinics during the COVID-19 crisis, teleophthalmology could make data more mobile and increase the geographical reach of specialist care ${ }^{42}$ reducing disruption to existing patient monitoring programmes. Store-andforward systems also passively amass a data bank of pathologies. In the Philadelphia Telemedicine Glaucoma Detection and Follow-up Study, ${ }^{42}$ of the 38 participants who were later diagnosed with visually significant cataracts on follow-up visits, $39.5 \%$ and $55.3 \%$ were originally classified as having "unreadable" and "abnormal" fundus images, respectively. Although the follow-up study targeted glaucoma patients, it was able to detect high rates of cataracts as a corollary effect.

\section{Diabetic retinopathy}

Similar results were noted in applying teleophthalmology to diagnosis and monitoring of diabetic retinopathy. ${ }^{15}$ A Chilean study using remote evaluation of digital images of patients' eyes noted increased screening coverage at the primary care level, more timely detection and decreased waiting times to see limited numbers of specialists. ${ }^{50}$ Another study noted that targeted referrals after using teleophthalmology as a screening tool increased adherence rates to hospital referrals and yield of diabetic retinopathy cases, ${ }^{21}$ thereby encouraging more efficient resource use. Date et al. reported a high level of accuracy in detecting and classifying diabetic retinopathy through remote review of teleretinal images. ${ }^{23}$ Although such application of teleophthalmology has to date been used as a screening tool, perhaps there is now incentive to trial such tools as alternatives to running physical clinics during the COVID-19 pandemic. 


\section{Macular degeneration}

In macular degeneration, optical coherence tomographic images have even been used for remote diagnostic evaluation. Results show remote diagnostic imaging to be equivalent to standard in-person examinations by retinal specialists for identifying patients requiring referral in a more timely fashion. ${ }^{35}$

\section{Cloud-based referral platforms}

A hospital in the UK implemented a cloud-based referral platform that used store-and-forward reviews by a consultant ophthalmologist to triage patients for referral appointments. The study noted a substantial reduction in unnecessary referrals, improved clinician productivity-9.2 minutes (optometrists) and 3.0 minutes (ophthalmologists) per patient - and that many conditions could be successfully identified by remote review, with the most common being age-related macular degeneration. ${ }^{52}$ These efficiency gains within ophthalmology clinics will allow for diversion of finite hospital resources and manpower to other specialties facing shortages in this COVID-19 crisis. Combined with the reduced risk of viral transmission that teleophthalmology affords both patients and healthcare workers, there is a compelling case for exploring teleophthalmology more vigorously.

\section{Real-time teleconferencing and real-time transmission of diagnostics}

Real-time (i.e. synchronous) teleconferencing is another key component of teleophthalmology. It allows for real-time communication that serves to bridge the physical doctor-patient distance, with the goal of simulating traditional face-to-face consultation. Its capability for synchronous clinical data transmission also allows for real-time communication of diagnosis and management plans. With the enactment of stay-home measures hindering in-person medical consults in this COVID-19 climate, online teleconsultations appear to hold great potential to meet the patients' clinical ophthalmological needs while eliminating clinic visits. ${ }^{45}$

Lions Outback Vision runs a state-wide teleophthalmological service linking patients in rural Western Australia to specialists based in the city, and in 2015, it performed a retrospective audit on the diagnostic outcomes of their optometry-facilitated teleophthalmology consult scheme. ${ }^{13}$ This scheme involved patient referrals from community optometrists for consultations with distant ophthalmologists via video conferencing software such as Skype. Over the course of 12 months, 709 patients were referred for teleconsultations, and 2 key benefits of teleophthalmology were noted: (1) decreased need for specialist outpatient appointment prior to surgical booking, and (2) cancellation of over 10 days of outreach clinic consultations, which allowed for allocation of clinic time to patients with more complex pathologies. Therefore, real-time teleconferencing shows the potential in handling ophthalmological conditions while greatly reducing face-to-face visits. ${ }^{26,32}$ It may come in handy as we cope with the increased call for social distancing in this epidemic.

Aside from its diagnostic capabilities, real-time teleconferencing has the potential to handle another important aspect of medicine-consent-taking. In a study comparing the quality of consent-taking for a refractive surgery between a telemedicine approach and a face-to-face discussion, majority of the 11,938 patients opted for telemedicine-assisted consent and were equally satisfied as their counterparts who chose face-to-face meeting. ${ }^{36}$ Surgeons can access the patients' clinical records and ophthalmological images online to support the consent-taking process, while incorporating audio-visual delivery of surgical counselling. This reduces the need for face-to-face interaction, which will be particularly important in this COVID-19 climate.

Telerehabiliation may also provide an avenue for longterm or postoperative management of ophthalmological needs.$^{30} \mathrm{~A}$ pilot study looked at low-vision telerehabilitation services conducted for 10 visually impaired adults. In-clinic providers assessed the patients' reading technique with their optical magnifier and administered the MNREAD test during videoconferencing sessions. ${ }^{41}$ Synchronous transmission of patients' video enabled assessment of reading speed and accuracy. Following that telerehabilitation session, providers considered the training provided via telerehabilitation to be similar in quality to that of in-clinic session. Application of real-time teleconferencing thus brings about possibilities in managing ophthalmological conditions, without the need for direct contact. This may be particularly useful for patients requiring rehabilitation-based management but find it inaccessible in this climate of reduced face-toface contact.

\section{Telemedicine in other specialties}

Restrictions on face-to-face interactions and social distancing measures put in place as part of the fight against the epidemic have forced physicians to search for alternatives to provide accessible healthcare. With a need to be met, there has been a growing interest in telemedicine in the various fields of medicine such as 
otolaryngology, ${ }^{56}$ endocrinology, ${ }^{57}$ oncology ${ }^{58}$ and others. One popular area of interest would be otolaryngology in which the use of smartphone applications has become more widespread in clinical practice. ${ }^{59}$ For instance, in a study preliminarily testing a smartphone-enabled otoscope (Mebird M9pro wireless otoscope), the physicians who examined patients' external auditory canal wirelessly managed to make real-time diagnosis and received excellent participation feedback. ${ }^{60}$ In dermatology, the Ohio State University Division of Dermatology implemented a WebEx virtual conference call system, which utilised a store-and-forward method, to perform inpatient telemedicine rounds. ${ }^{61}$ It was received with overwhelmingly positive response and increased clinical efficiency.

Overall, telemedicine is being increasingly explored by multiple specialisations during this pandemic as the healthcare sector seeks to cope with the limitations imposed. ${ }^{29}$

\section{Limitations of teleophthalmology}

\section{Efficiency}

The efficiency gains of adopting teleophthalmology discussed in this review may be eroded by teleophthalmology-specific inefficiencies.

In several studies, poor image ${ }^{34}$ and video quality require patients to be recalled for reassessment or a classical face-to-face examination; ${ }^{82}$-one study recorded $6.5 \%$ of its videos as having poor quality. ${ }^{22}$ Images lost to technical errors require the same response. Administrative difficulties in recalling patients for reassessment may result in greater time inefficiencies. Furthermore, in store-and-forward teleophthalmology, each patient incurs a time lag between assessment and diagnosis, while their images are sent for review. Important diagnoses may thus be delayed. ${ }^{62}$

Teleophthalmology also places an additional administrative burden on staff for tasks such as data entry and upload. Only a handful of studies have accounted for additional administrative load, ${ }^{47,52}$ and no study has directly compared total time taken for teleophthalmology with that for face-to-face examinations, or proven a quantifiable reduction in overall manpower required.

\section{Software requirements}

Although real-time teleconsultations could potentially eliminate clinic visits, this is largely dependent on powerful software transmissions and processing speeds to simulate clinic consultations. In the study implementing virtual medical retina clinic, efficiency was hindered owing to the requirement of several software programs running in parallel. ${ }^{45}$

It has been suggested that artificial intelligence (AI) tools such as deep learning may help overcome various inefficiencies that teleophthalmology faces. ${ }^{57}$ For instance, artificial learning algorithms may assist with clinical image processing more accurately, potentially translating into enhanced diagnostic efficiency. In another example, AI-based chatbots can provide patient-initiated interactions and a triage of the patients' symptoms, followed by initial advice of self-care, while necessary cases could be referred to a clinician for further evaluation through either virtual or physical means. ${ }^{63}$ Granted, it may take time to amass data and train the machine learning program. ${ }^{64,65}$ However, once trained to sufficient accuracy, it may prove an invaluable tool.

\section{Liabilities}

Teleophthalmology could potentially increase liabilities for healthcare workers and hospitals.

Despite yielding results comparable to classical examination methods, fears that teleophthalmology will miss or misinterpret crucial diagnoses remain prevalent. Given that many teleophthalmology technologies have not yet been trialled for their safety as anything more than first-pass triaging tools,${ }^{49}$ implementing teleophthalmology may increase liability exposure. Confidence of medical practitioners in teleophthalmology needs to be garnere ${ }^{20,46}$ and is unlikely to be gained until a robust legal liability framework is developed.

In one study, patients expressed concerns over data privacy and hesitated to use teleophthalmology. ${ }^{41}$ Liabilities would be incurred in the event of hacking or data leaks. These concerns are, however, mitigated by data encryption and compliance to various data security and health insurance accountability acts. ${ }^{42}$ Given the evolving use of digital tools for COVID-19 contact tracing, the focus on securing patients' data privacy is greater than ever. ${ }^{66}$

Lastly, an asynchronous teleconsultation system creates a digital trace, which may give rise to medicolegal issues. Careful phrasing of information and detailed consent taking will be needed to ensure informed consent, given that data in storage could be used for further diagnostic, management or research purposes that differ from the original intention of screening. ${ }^{41}$ However, Mastropasqua et al. highlight that this could also be advantageous. Among 3,679 patients with gradable mydriatic fundus images originally intended for grading diabetic retinopathy, a wide range of conditions such as age-related macular degeneration and hypertensive 
retinopathy could be incidentally detected. ${ }^{47}$ Therefore, patient data can be revisited at a later date to screen for other pathologies, without requiring additional patient consultations.

\section{Limitations of current literature review}

This study has several limitations. As only one database, namely PubMed, was searched, this may potentially introduce selection bias to the articles we have included for review. As only studies published in English were included, relevant articles in other languages would have been excluded.

Next, although both qualitative and quantitative studies were reviewed, most of the studies were qualitative, thereby potentially limiting our results scope. The MMAT tool was used to appraise the methodological quality of these mixed methods of study, but no quantitative analysis could be performed to unify the screened results.

Furthermore, as outcomes discussed mainly involved broad overviews of teleophthalmology, there was a lack of consistent outcome measures in the studies. Thus, no quantifiable data were measured to prove the reported advantages of teleophthalmology, such as increased efficiency. Publication bias may have also favoured the reporting of virtues of teleophthalmology over its limitations, especially in quantitative descriptive studies.

Lastly, both the COVID-19 situation and teleophthalmology as a concept are rapidly evolving. Therefore, our results are only accurate up to the date of screening and may not reflect more recent changes.

\section{CONCLUSION}

Teleophthalmology has been implemented in multiple aspects of patient care, ranging from diagnostic evaluations to therapeutic management. Multiple studies have reported its potential in reducing doctor-patient contact requirement and enhancing care provision efficiency, although none has quantified the costeffectiveness of teleophthalmology over traditional means of healthcare. Despite limitations in its current form, teleophthalmology may be a viable option in tackling the medical limitations imposed by the COVID-19 pandemic.

\section{REFERENCES}

1. World Health Organization. Coronavirus (COVID-19) outbreak. Available at: https://www.who.int/westernpacific/emergencies/ covid-19. Accessed on 27 April 2020.

2. Lauer SA, Grantz KH, Bi Q, et al. The incubation period of coronavirus disease 2019 (COVID-19) from publicly reported confirmed cases: Estimation and application. Ann Intern Med 2020;172:577-82.
3. Lai THT, Tang EWH, Chau SKY, et al. Stepping up infection control measures in ophthalmology during the novel coronavirus outbreak: an experience from Hong Kong. Graefes Arch Clin Exp Ophthalmol 2020;258:1049-55.

4. Ohannessian R, Duong TA, Odone A. Global telemedicine implementation and integration within health systems to fight the COVID-19 pandemic: A call to action. JMIR Public Health Surveill 2020;6:e18810.

5. Hong N, Yu W, Xia J, et al. Evaluation of ocular symptoms and tropism of SARS-CoV-2 in patients confirmed with COVID-19. Acta Ophthalmol 2020;98:e649-55.

6. Seah I, Su X, Lingam G. Revisiting the dangers of the coronavirus in the ophthalmology practice. Eye (Lond) 2020;34:1155-7.

7. Xie X, Li Y, Chwang ATY, et al. How far droplets can move in indoor environments - revisiting the Wells evaporation-falling curve. Indoor Air 2007; 17:211-25.

8. Khor WB, Yip L, Zhao P, et al. Evolving practice patterns in Singapore's public sector ophthalmology centers during the COVID-19 pandemic. Asia Pac J Ophthalmol (Phila) 2020;9:285-90.

9. Jun ISY, Hui KKO, Songbo PZ. Perspectives on coronavirus disease 2019 control measures for ophthalmology clinics based on a Singapore center experience. JAMA Ophthalmol 2020;138:435-6.

10. Institute of Medicine (US) Committee on Evaluating Clinical Applications of Telemedicine. Telemedicine: A Guide to Assessing Telecommunications in Health Care. Washington (DC): National Academies Press (US); 1996.

11. Ting DS, Gunasekeran DV, Wickham L, et al. Next generation telemedicine platforms to screen and triage. $\mathrm{Br} \mathrm{J}$ Ophthalmol 2020;104:299-300.

12. Maa AY, Patel S, Chasan JE, et al. Retrospective evaluation of a teleretinal screening program in detecting multiple nondiabetic eye diseases. Telemed J E Health 2017;23:41-8.

13. Bartnik SE, Copeland SP, Aicken AJ, et al. Optometry-facilitated teleophthalmology: An audit of the first year in Western Australia. Clin Exp Optom 2018;101:700-3

14. Kozak I, Payne JF, Schatz P, et al. Teleophthalmology image-based navigated retinal laser therapy for diabetic macular edema: A concept of retinal telephotocoagulation. Graefes Arch Clin Exp Ophthalmol 2017;255:1509-13.

15. Yaslam M, Al Adel F, Al-Rubeaan K, et al. Non-mydriatic fundus camera screening with diagnosis by telemedicine for diabetic retinopathy patients with type 1 and type 2 diabetes: A hospital-based cross-sectional study. Ann Saudi Med 2019;39:328-36.

16. Giorgis AT, Alemu AM, Arora S, et al. Results from the first teleglaucoma pilot project in Addis Ababa, Ethiopia. J Glaucoma 2019;28:701-7.

17. Tricco AC, Lillie E, Zarin W, et al. PRISMA Extension for Scoping Reviews (PRISMA-ScR): Checklist and explanation. Ann Intern Med 2018;169:467-73.

18. Hong QN, Fàbregues S, Bartlett G, et al. The Mixed Methods Appraisal Tool (MMAT) version 2018 for information professionals and researchers. Educ Inf 2018;34:285-91.

19. Daruich A, Martin D, Bremond-Gignac D. Ocular manifestation as first sign of Coronavirus Disease 2019 (COVID-19): Interest of telemedicine during the pandemic context. J Fr Ophtalmol 2020; 43:389-91.

20. Liu Y, Zupan NJ, Swearingen R, et al. Identification of barriers, facilitators and system-based implementation strategies to increase teleophthalmology use for diabetic eye screening in a rural US primary care clinic: A qualitative study. BMJ Open 2019; 9:e022594. 
21. Joseph S, Kim R, Ravindran RD, et al. Effectiveness of teleretinal imaging-based hospital referral compared with universal referral in identifying diabetic retinopathy: A cluster randomized clinical trial. JAMA Ophthalmol 2019;137:786-92.

22. Laurent C, Hong SC, Cheyne KR, et al. The detection of spontaneous venous pulsation with smartphone video ophthalmoscopy. Clin Ophthalmol 2020;14:331-7.

23. Date RC, Shen KL, Shah BM, et al. Accuracy of detection and grading of diabetic retinopathy and diabetic macular edema using teleretinal screening. Ophthalmol Retina 2019;3:343-9.

24. Wisse RPL, Muijzer MB, Cassano F, et al. Validation of an independent Web-based tool for measuring visual acuity and refractive error (the manifest versus online refractive evaluation trial): Prospective open-label noninferiority clinical trial. J Med Internet Res 2019;21:e14808.

25. Maa AY, Medert CM, Lu X, et al. Diagnostic accuracy of Technology-based Eye Care Services: The Technology-based Eye Care Services Compare trial part I. Ophthalmology 2020; 127:38-44

26. Roelofs K, Weis E. Tele-oncology: A validation study of choroidal and iris nevi. Ocul Oncol Pathol 2019;5:298-302.

27. Chandrasekaran S, Kass W, Thangamathesvaran L, et al. Teleglaucoma vs clinical evaluation: The New Jersey Health Foundation prospective clinical study. J Telemed Telecare 2020;26:536-44.

28. Phanphruk W, Liu Y, Morley K, et al. Validation of StrabisPIX, a mobile application for home measurement of ocular alignment. Transl Vis Sci Technol 2019;8:9.

29. Tsapakis S, Papaconstantinou D, Diagourtas A, et al. Homebased visual field test for glaucoma screening comparison with Humphrey perimeter. Clin Ophthalmol 2018;12:2597-606.

30. Young K, Gupta A, Palacios R. Impact of telemedicine in pediatric postoperative care. Telemed J E Health 2019;25:1083-9.

31. Lapere S, Weis E. Tele-ophthalmology for the monitoring of choroidal and iris nevi: A pilot study. Can J Ophthalmol 2018; $53: 471-3$

32. Gonzalez F, Cimadevila B, Garcia-Comesaña J, et al. Telephone consultation in primary care. J Health Organ Manag 2018;32:321-37.

33. Bursztyn L, Woodward MA, Cornblath WT, et al. Accuracy and reliability of a handheld, nonmydriatic fundus camera for the remote detection of optic disc edema. Telemed J E Health 2018; 24:344-50

34. Maka E, Kovács G, Imre L, et al. The validity of telemedicine-based screening for retinopathy of prematurity in the Premature Eye Rescue Program in Hungary. J Telemed Telecare 2019;1357633X19880113.

35. Hadziahmetovic M, Nicholas P, Jindal S, et al. Evaluation of a remote diagnosis imaging model vs dilated eye examination in referable macular degeneration. JAMA Ophthalmol 2019;137:802-8.

36. Schallhorn SC, Hannan SJ, Teenan D, et al. Informed consent in refractive surgery: In-person vs telemedicine approach. Clin Ophthalmol 2018;12:2459-70.

37. Odden JL, Khanna CL, Choo CM, et al. Telemedicine in long-term care of glaucoma patients. J Telemed Telecare 2020;26:92-9.

38. Amparo F, Dana R. Web-based longitudinal remote assessment of dry eye symptoms. Ocul Surf 2018;16:249-53.

39. Starr MR, Barkmeier AJ, Engman SJ, et al. Telemedicine in the management of exudative age-related macular degeneration within an integrated health care system. Am J Ophthalmol 2019;208:206-10.

40. Hark LA, Myers JS, Ines A, et al. Philadelphia Telemedicine Glaucoma Detection and Follow-up Study: confirmation between eye screening and comprehensive eye examination diagnoses. Br J Ophthalmol 2019;103:1820-6.
41. Bittner AK, Yoshinaga P, Bowers A, et al. Feasibility of telerehabilitation for low vision: Satisfaction ratings by providers and patients. Optom Vis Sci 2018;95:865-72.

42. Hark LA, Adeghate J, Katz LJ, et al. Philadelphia Telemedicine Glaucoma Detection and Follow-up Study: Cataract classifications following eye screening. Telemed J E Health2020;26:992-1000.

43. Safi S, Ahmadieh $\mathrm{H}$, Katibeh $\mathrm{M}$, et al. Modeling a telemedicine screening program for diabetic retinopathy in Iran and implementing a pilot project in Tehran suburb. J Ophthalmol 2019;2019:2073679.

44. Modjtahedi BS, Chu K, Luong TQ, et al. Two-year outcomes of a pilot glaucoma suspect telemedicine monitoring program. Clin Ophthalmol Auckl NZ 2018;12:2095-102.

45. Kortuem K, Fasler K, Charnley A, et al. Implementation of medical retina virtual clinics in a tertiary eye care referral centre. Br J Ophthalmol 2018;102:1391-5.

46. Host BK, Turner AW, Muir J. Real-time teleophthalmology video consultation: An analysis of patient satisfaction in rural Western Australia. Clin Exp Optom 2018;101:129-34.

47. Mastropasqua L, Perilli R, D'Aloisio R, et al. Why miss the chance? Incidental findings while telescreening for diabetic retinopathy. Ophthalmic Epidemiol 2020;27:237-45.

48. Strul S, Zheng Y, Gangaputra S, et al. Pediatric diabetic retinopathy telescreening. J AAPOS 2020;24:10.e1-5.

49. Martinez JA, Parikh PD, Wong RW, et al. Telemedicine for diabetic retinopathy screening in an urban, insured population using fundus cameras in a primary care office setting. Ophthalmic Surg Lasers Imaging Retina 2019;50:e274-7

50. Avendaño-Veloso A, Parada-Hernández F, González-Ramos R, et al. Teleophthalmology: A strategy for timely diagnosis of sight-threatening diabetic retinopathy in primary care, Concepción, Chile. Int J Ophthalmol 2019;12:1474-8.

51. Grau E, Horn F, Nixdorff U, et al. OCT and IOP findings in a healthy worker cohort: Results from a teleophthalmic study in occupational medicine. Graefes Arch Clin Exp Ophthalmol 2019;257:2571-8.

52. Kern C, Fu DJ, Kortuem K, et al. Implementation of a cloud-based referral platform in ophthalmology: Making telemedicine services a reality in eye care. Br J Ophthalmol 2020;104:312-7.

53. Afshar AR, Oldenburg CE, Stewart JM. A novel hybrid fixed and mobile ultra-widefield imaging program for diabetic teleretinopathy screening. Ophthalmol Retina 2019;3:576-9.

54. Kang L, Li Y, Hu S, et al. The mental health of medical workers in Wuhan, China dealing with the 2019 novel coronavirus. Lancet Psychiatry 2020;7:e14.

55. Wong IYH, Ni MY, Wong IOL, et al. Saving sight in China and beyond: The Lifeline Express model. BMJ Glob Health 2018; 3:e000766.

56. Pollock K, Setzen M, Svider PF. Embracing telemedicine into your otolaryngology practice amid the COVID-19 crisis: An invited commentary. Am J Otolaryngol 2020;41:102490.

57. Mohan V. COVID-19 and diabetes - a view from India. J Diabetes Sci Technol 2020;14:760-1.

58. Lewis GD, Hatch SS, Wiederhold LR, et al. Long-term institutional experience with telemedicine services for radiation oncology: A potential model for long-term utilization. Adv Radiat Oncol 2020;5:780-2

59. Tabanfar R, Chan HHL, Lin V, et al. Development and face validation of a Virtual Reality Epley Maneuver System (VREMS) for home Epley treatment of benign paroxysmal positional vertigo: A randomized, controlled trial. Am J Otolaryngol 2018;39:184-91 
60. Meng X, Dai Z, Hang C, et al. Smartphone-enabled wireless otoscope-assisted online telemedicine during the COVID-19 outbreak. Am J Otolaryngol 2020;41:102476.

61. Rismiller K, Cartron AM, Trinidad JCL. Inpatient teledermatology during the COVID-19 pandemic. J Dermatol Treat 2020;31:441-3.

62. Safdari R, Langarizadeh M, Ramezani A, et al. Development of a store-and-forward telescreening system of diabetic retinopathy: Lessons learned from Iran. J Diabetes Metab Disord 2018;17:31-6.

63. Burki T. GP at hand: a digital revolution for health care provision? Lancet 2019;394:457-60.
64. Kapoor R, Walters SP, Al-Aswad LA. The current state of artificial intelligence in ophthalmology. Surv Ophthalmol 2019; 64:233-40.

65. Ahn JM, Kim S, Ahn KS, et al. A deep learning model for the detection of both advanced and early glaucoma using fundus photography. PloS One 2018;13:e0207982.

66. Ekong I, Chukwu E, Chukwu M. COVID-19 mobile positioning data contact tracing and patient privacy regulations: Exploratory search of global response strategies and the use of digital tools in Nigeria. JMIR MHealth UHealth 2020;8:e19139. 